\title{
Plasmonic Substrates Do Not Promote Vibrational Energy Transfer at Solid-Liquid Interfaces
}

\author{
Kraack, Jan Philip ; Sévery, Laurent ; Tilley, S David ; Hamm, Peter
}

\begin{abstract}
Intermolecular vibrational energy transfer in monolayers of isotopically mixed rhenium carbonyl complexes at solid-liquid interfaces is investigated with the help of ultrafast 2D Attenuated Total Reflectance Infrared (2D ATR IR) spectroscopy in dependence of plasmonic surface enhancement effects. Dielectric and plasmonic materials are used to demonstrate that plasmonic effects have no impact on the vibrational energy transfer rate in a regime of moderate IR surface enhancement (enhancement factors up to ca. 30). This result can be explained with the common image-dipole picture. The vibrational energy transfer rate thus can be used as a direct observable to determine intermolecular distances on surfaces, regardless of their plasmonic properties.
\end{abstract}

DOI: https://doi.org/10.1021/acs.jpclett.7b02855

Posted at the Zurich Open Repository and Archive, University of Zurich

ZORA URL: https://doi.org/10.5167/uzh-146994

Journal Article

Accepted Version

Originally published at:

Kraack, Jan Philip; Sévery, Laurent; Tilley, S David; Hamm, Peter (2017). Plasmonic Substrates Do Not Promote Vibrational Energy Transfer at Solid-Liquid Interfaces. Journal of Physical Chemistry Letters, 9(1):49-56.

DOI: https://doi.org/10.1021/acs.jpclett.7b02855 


\section{Plasmonic Substrates Do Not Promote Vibrational Energy Transfer at Solid-Liquid Interfaces}

Jan Philip Kraack*, Laurent Sévery, S. David Tilley, and Peter Hamm

Department of Chemistry, University of Zurich, Winterthurerstrasse 190, CH-8057 


\section{Abstract}

Intermolecular vibrational energy transfer in monolayers of isotopically mixed rhenium carbonyl complexes at solid-liquid interfaces is investigated with the help of ultrafast 2D Attenuated Total Reflectance Infrared (2D ATR IR) spectroscopy in dependence of plasmonic surface enhancement effects. Dielectric and plasmonic materials are used to demonstrate that plasmonic effects have no impact on the vibrational energy transfer rate in a regime of moderate IR surface enhancement (enhancement factors up to ca. 30). This result can be explained with the common image-dipole picture. The vibrational energy transfer rate thus can be used as a direct observable to determine intermolecular distances on surfaces, regardless of their plasmonic properties.

\section{TOC Graphic}

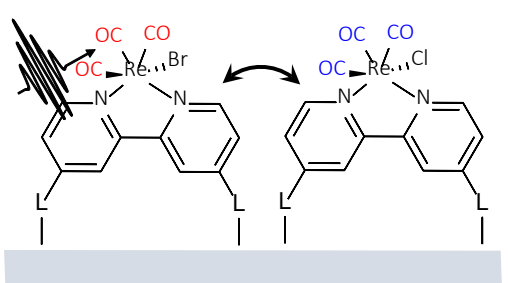

\section{Keywords:}

Metal carbonyl complexes, self-assembled monolayers, vibrational energy transfer, surface vibrational spectroscopy, 2D ATR IR spectroscopy, plasmonic spectroscopy, surface-enhanced infrared spectroscopy. 
Interactions of molecules at surfaces are extremely important in different fields of current research, such as heterogeneous catalysis, artificial light-harvesting or molecular sensing and recognition. However, it is challenging to directly observe the dynamics of interactions spectroscopically. This is particularly true for molecules that do not form chemical bonds, through which mutual interactions could occur, but rather interact "through space" in an electrostatic way, e.g. by dipole-dipole coupling. One of the most prominent ways to witness such interactions is energy transfer between different chemical entities. ${ }^{1-4}$ This effect is well established in the field of electronic spectroscopy with visible light (Förster energy transfer), as well as for NMR spectroscopy (NOESY), where energy transfer has been observed and used to elucidate structural properties of various samples. ${ }^{1-3,5,6}$ For vibrational spectroscopy in the mid-infrared (IR) range, intermolecular vibrational energy transfer through space is, however, much less frequently observed, but still holds great promise to facilitate structural investigations of samples, such as relative molecular distances and orientations. ${ }^{4,7-15}$ It is therefore strongly desirable to develop experimental strategies, through which vibrational energy transfer can be engineered.

The vibrational energy transfer rate $\mathrm{k}_{\mathrm{ET}}$ is commonly described by a mechanism based on the transition dipole coupling $\mathrm{V}_{\mathrm{DD}}$ (Eq. 1), whose dominant factors are the mutual distance of a donor and an acceptor $r$, the contributing transition dipole moments $\mu_{D}$ and $\mu_{A}$ of donor and acceptor, respectively (which we will assume to be the same $\mu_{D}=\mu_{A}=\mu$ ), and a geometry factor $\kappa$ describing the orientation of the transition dipoles relative to a vector connecting the two molecules. All factors enter with a strongly nonlinear dependence into the vibrational energy transfer rate $\mathrm{k}_{\mathrm{ET}}:^{:-4,11,13}$

$$
\mathrm{k}_{\mathrm{ET}} \propto \mathrm{V}_{\mathrm{DD}}^{2}=\kappa^{2} \frac{\mu_{\mathrm{D}}^{2} \mu_{\mathrm{A}}^{2}}{\mathrm{r}^{6}}=\kappa^{2} \frac{\mu^{4}}{\mathrm{r}^{6}}
$$

in close analogy to NOESY in NMR ${ }^{5}$, or Förster energy transfer in fluorescence spectroscopy. ${ }^{6}$ In order to render the intermolecular distance small, immobilization of molecules at surfaces is a beneficial approach, since packing densities in molecular monolayers are generally high with sub-nanometer intermolecular distances. ${ }^{16,17}$ Thus, molecular monolayers are prime candidates to observe vibrational energy transfer, and quite some experimental effort went into this direction recently. ${ }^{8,18-25}$ We have shown in Ref. ${ }^{8}$ that in addition to a short distance, a large transition dipole $\mu$ is required to compete with the generally short lifetime of vibrational transitions. As of today, intermolecular vibrational energy transfer for a molecular monolayer has been observed only for a metal-carbonyl complex, owing to its exceptionally large transition dipole in combination with a comparably long lifetime. ${ }^{8}$ In essence, the equivalent of a "Förster radius" for vibrational transitions (i.e., the distance at which the vibrational energy transfer rate equals the inverse lifetime) is small ( 3-4 $\AA$ ) even for metal-carbonyls that exhibit a very high transition dipole already. This small value limits the versatility of the approach, and even higher transition dipoles would be desirable. ${ }^{26-28}$ 
In that regard, it is well known that the IR absorption cross section of molecules on metallic surfaces are often strongly enhanced by plasmonic effects, either on rough metal surface or even more so with specifically designed nano-structures. ${ }^{29-32}$ The absorption cross section $\mathrm{A}$ of a vibrational transition scales as:

$$
\mathrm{A} \propto \mu_{\mathrm{eff}}^{2}
$$

where $\mu_{\text {eff }}$ is an effective, potentially enhanced transition dipole. Considering Eqs. 1 and 2, one might therefore assume that vibrational energy transfer is enhanced as well on plasmonic surfaces, provided that the transition dipole moments relevant for both effects are the same. The aim of this paper is to test whether that is indeed the case.

To that end, we employ a surface-sensitive variant of 2D IR spectroscopy, ${ }^{33-35}$ since this allows direct observation of vibrational energy transfer through the identification of cross peaks in the signals. Specifically, we use 2D ATR IR spectroscopy, which has been introduced recently as a new and very flexible technique for surface vibrational characterization of adsorbates in different environments. ${ }^{35-37}$ The information content in 2D ATR IR spectra is analogous to that of "classic" 2D IR spectroscopy in transmission geometry ${ }^{33,34}$, but rather exploits evanescent waves at the interface of an ATR element to excite and probe the sample.

The molecule under study is a metal-carbonyl complex, $\operatorname{Re}\left(4,4^{\prime}\right.$-di-linker-2,2'-bipyridine $)(\mathrm{CO})_{3} \mathrm{Cl}$ $\left(\operatorname{Re}(\mathrm{CO})_{3} \mathrm{Cl}\right.$, see Figure 1). The same $e^{24,25,38-41}$ or very similar metal carbonyl complexes ${ }^{18,42-46}$ on different types of surfaces have already been investigated quite intensively recently with the help of 2D IR and 2D-SFG spectroscopy. The importance of surface heterogeneity, $24,25,38,39,41,44$ the intramolecular dynamics within the immobilized molecule, ${ }^{40}$ as well as the coupling to the surface ${ }^{42,43}$ have been pointed out. Moreover, vibrational couplings have been observed between different subbands when the complexes aggregate on the surface. ${ }^{24,25}$ Form these works, it is well established that the adsorbates dynamics strongly depend on subtle properties of the substrate. 


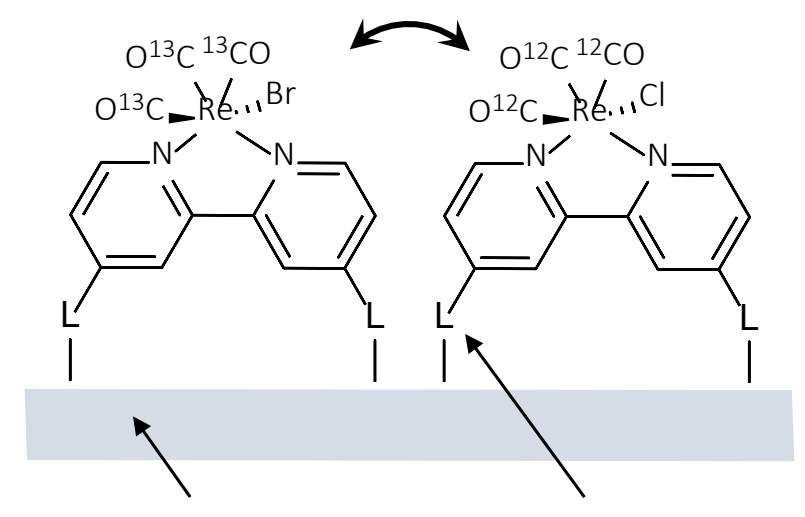

Figure 1. Sample system as used in this study. Sample molecules $\left(\operatorname{Re}\left(4,4^{\prime}-\right.\right.$ di-linker-2,2'bipyridine $)\left({ }^{13} \mathrm{CO}\right)_{3} \mathrm{Br}\left(\operatorname{Re}\left({ }^{13} \mathrm{CO}\right){ }_{3} \mathrm{Br}\right)$ and $\operatorname{Re}\left(4,4^{\prime}\right.$-di-linker-2, $2^{\prime}$-bipyridine $)\left({ }^{12} \mathrm{CO}\right){ }_{3} \mathrm{Cl}\left(\operatorname{Re}\left({ }^{12} \mathrm{CO}\right){ }_{3} \mathrm{Cl}\right)$ are covalently adsorbed as about 1:1 mixtures on titanium dioxide $\left(\mathrm{TiO}_{2}\right)$, Indium-Tin-Oxide (ITO) and Gold (Au) surfaces. Depending on the type of surface, the linker $\mathrm{L}$ is a carboxyl group for oxide surfaces $\left(\mathrm{TiO}_{2}\right.$ and ITO), or a methyl thioether (S-Me) for $\mathrm{Au}$.

A detailed sketch of the complete sample system under investigation in the present study is shown in Figure 1. Mixed (1:1) monolayers with $\operatorname{Re}\left(4,4^{\prime}\right.$-di-linker-2,2'-bipyridine $)\left({ }^{13} \mathrm{CO}\right){ }_{3} \mathrm{Br}$ $\left(\operatorname{Re}\left({ }^{13} \mathrm{CO}\right){ }_{3} \mathrm{Br}\right)$ and $\operatorname{Re}\left(4,4^{\prime}\right.$-di-linker-2,2'-bipyridine $)\left({ }^{12} \mathrm{CO}\right){ }_{3} \mathrm{Cl}\left(\operatorname{Re}\left({ }^{12} \mathrm{CO}\right){ }_{3} \mathrm{Cl}\right)$ head groups are covalently adsorbed to different types of surfaces, which are deposited on the reflecting plane of a $\mathrm{CaF}_{2}$ ATR prism. The molecules are attached to the surfaces at the 4,4'-positions of the bipyridine ligands via different linkers ("L", Figure 1), depending on the type of the surface. That is, two carboxyl groups at the bipyridine ligands are used for immobilization in case of oxide surfaces ( $\mathrm{TiO}_{2}$ and ITO), whereas two methyl thioether groups are employed in case of a metal surface (Au). In the latter case, we use thioethers rather than thiols to prevent dimerization of the molecules in solution by oxidative formation of disulfide bonds. The employed Re-carbonyl molecules exhibit several prominent benefits regarding their use as reporters of vibrational energy transfer. First, the transition dipole of the totally symmetric carbonyl-stretching vibration is large, as evidenced by the high absorption coefficient of $\approx 3400 \mathrm{M}^{-1} \mathrm{~cm}^{-1}$. This aspect facilitates a reasonable absorption from only monolayer thin samples at the interface (Eq. 2) as well as a reasonably fast vibrational energy transfer rate (Eq. 1). Second, the molecules form stable bonds to the surface due to their two easily accessible functional groups for immobilization and thus feature a very low degree of structural flexibility. Third, the molecules exhibit a considerable timescale for vibrational relaxation with lifetimes larger than 20 ps, which allows a long temporal observation window (about $60 \mathrm{ps}$ ). Fourth, the totally symmetric $A^{\prime}(1)$ stretch vibration of 
the carbonyl ligands is located in an experimentally well accessible spectral region around $2000 \mathrm{~cm}^{-1}$ and modes from different molecules can easily be made spectroscopically distinguishable by use of ${ }^{13} \mathrm{C}$ isotope substitution of the carbonyl groups. ${ }^{8}$ That is, the $\mathrm{A}^{\prime}(1)$ vibration of $\operatorname{Re}\left({ }^{12} \mathrm{CO}\right){ }_{3} \mathrm{Cl}$ absorbs at about $2025 \mathrm{~cm}^{-1}$, whereas that of $\operatorname{Re}\left({ }^{13} \mathrm{CO}\right){ }_{3} \mathrm{Br}$ is located at about $1976 \mathrm{~cm}^{-1}$. We have recently shown that vibrational energy transfer can be observed between these two modes when the sample is immobilized on ITO. ${ }^{8}$

In the present study, the same molecular system has been immobilized on a series of different substrate layers, representing different levels of plasmonic properties. As the one extreme case, $\mathrm{TiO}_{2}$ is a purely dielectric material and the incident IR light interacts with the adsorbate molecules only but cannot polarize the $\mathrm{TiO}_{2}$ layer. Indeed we measured an "enhancement factor" of $\mathrm{EF}=1$, i.e. no enhancement (Fig. SI 1 (e) and (f)) for $\mathrm{TiO}_{2}$, which thus acts exclusively as a layer for sample immobilization at the interface. Conversely, metal layers represent the other extreme case, since they are strongly polarizable. ${ }^{29,31,32,47}$ Particularly Au layers of up to a few nanometre thickness have been shown previously to yield a significant surface enhancement in 2D ATR IR spectroscopy, which stems from a polarization of the metal substrate by the incident IR light along with possible other enhancement mechanisms such as chemical contributions. ${ }^{48,49}$ For our specific sample preparation, we measured an enhancement factor of about EF = 30 (Fig. SI 1 (a) and (b)). Between the two extreme cases, Indium-Tin-Oxide (ITO) is chosen as a material exhibiting electrical conductivity ${ }^{50}$ and a high mobility of electrons, which has been reported to facilitate weak plasmonic properties. ${ }^{51-53}$ For our particular sample preparation, we have measured an enhancement factor of about EF=2 (Fig. SI 1 (c) and (d)). Besides orientational effects by aligning the molecules on the surface, ${ }^{35,48,54}$ this small enhancement factor ITO might indeed reflect a weak plasmonic signal enhancement; nevertheless ITO is certainly more on the side of a dielectric than on the side of a metal. Comparing the dynamics of vibrational energy transfer on these surfaces with otherwise as identical as possible sample conditions allows us to study the impact of substrate polarizability and plasmonic surface enhancement on the vibrational energy transfer mechanism.

Figure 2 shows 2D ATR IR spectra of the mixed monolayer sample system $\operatorname{Re}\left({ }^{13} \mathrm{CO}\right){ }_{3} \mathrm{Br} / \operatorname{Re}\left({ }^{12} \mathrm{CO}\right)_{3} \mathrm{Cl}$ for two representative population delays (i.e. $0.5 \mathrm{ps}$ and $21 \mathrm{ps}$ ) for the three different surface materials, i.e., Figure 2 (a)/(b) for $\mathrm{TiO}_{2}$, (c)/(d) for ITO layers, and (e)/(f) for Au. All samples have been incubated with methanol during the experiments. The measurements for ITO are in principle analogous to Ref. ${ }^{8}$, but have been re-measured for consistency reasons. For all samples, the 2D ATR IR spectra at early population delays ( 0.5 ps) exhibit ground state bleach (blue) and excited state absorption signals (red) on the diagonal line. The signals are strongly elongated along the diagonal, reflecting the surface heterogeneity and a distribution of local solvation environments of the 
adsorbates. ${ }^{35}$ As the population time is increased to a few tens of picoseconds, weak cross peaks start to appear for each sample system. These cross peaks originate from vibrational energy transfer between the different molecules at the interface. ${ }^{8}$ Interestingly, the relative intensity between the cross peaks and the diagonal peaks is very similar for the $\mathrm{TiO}_{2} / \mathrm{ITO}$ samples and slightly smaller for the Au layer.
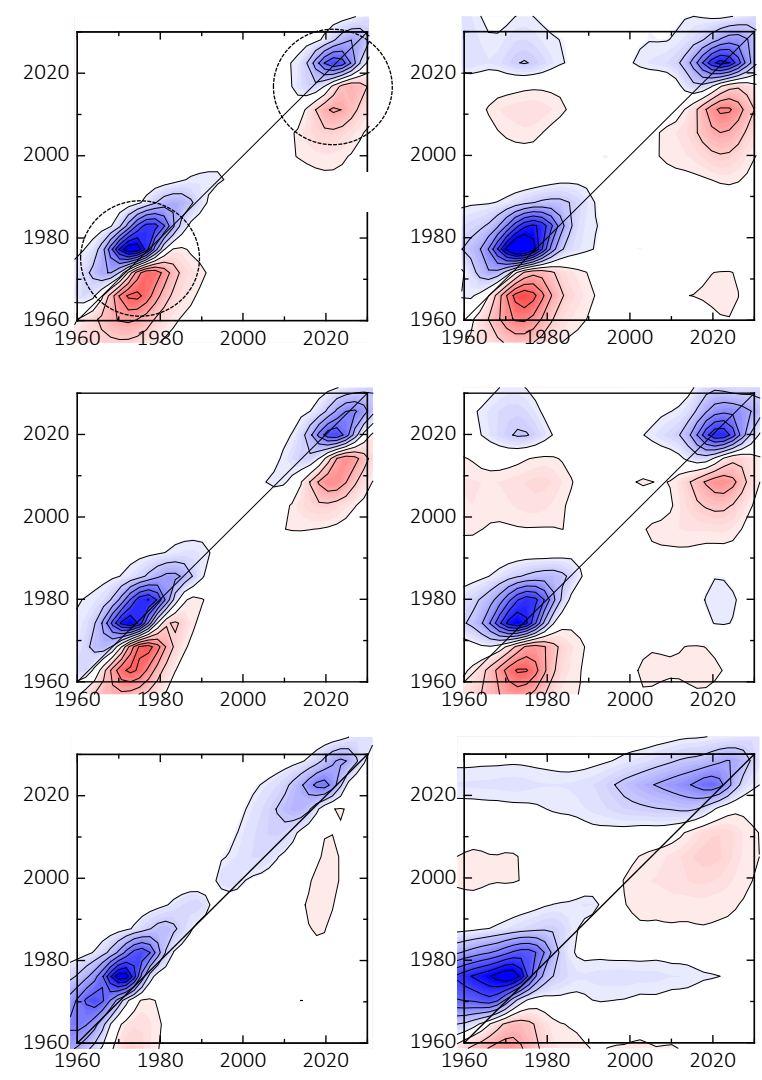

Figure 2. 2D ATR IR spectra from the $A^{\prime}(1)$ carbonyl stretching region at different waiting times ( $0.5 \mathrm{ps}$ and $21 \mathrm{ps}$ ) for the different sample systems shown in Figure 1 and immersed in $\mathrm{MeOH}$. Samples adsorbed to (a) and (b) $\mathrm{TiO}_{2}$, (c) and (d) ITO, (e) and (f) Au surfaces are compared. The circles in (a) indicate the different contributions for the two Rhenium complexes. Blue and red signals represent ground state bleach and excited state absorption signals, respectively. Cross peaks appear with increasing population delays, indicating vibrational energy transfer between the molecules. Signals are shown normalized to maximum ground state bleach intensity at each population delay to facilitate comparison.

To elucidate the detailed kinetics of vibrational energy transfer, we measured 2D ATR IR spectra at a full series of population delays that covers multiples of the vibrational lifetime of the complexes. Figure $3(a)-(c)$ shows the temporal evolution of both diagonal (circles) as well as cross peaks (triangles) up to a population delay of 60 ps. For all samples, the diagonal peaks decay monotonically, whereas the cross peak signals initially increase, peak at population delays between 13 ps and 25 ps, 
and then decay again. We observe similar maximum cross peak intensity and similar delays for the appearance of the cross peak maxima for $\mathrm{TiO}_{2}$ and ITO layers (Figure 3 (a) and (b)), whereas the intensity of the cross peaks is lower by approximately a factor of two in case of the Au layers and the cross peak maximum is significantly delayed (Figure 3 (c)). The data are fitted (red lines) with the kinetic model depicted in Figure $3(\mathrm{~d}) .{ }^{8}$ In that scheme, both molecules can be excited independently (stars) and can afterwards either transfer the excess vibrational energy to non-excited neighbouring molecules with time constant $\tau_{\mathrm{ET}}$, or undergo vibrational relaxation with time constants $\tau_{1,2}$. For all three systems, we observe that vibrational relaxation occurs in a bi-exponential manner, with time constants of $2-3 \mathrm{ps}$ (fast) and $20-21 \mathrm{ps}$ (slow) and amplitude ratios between these two contributions of about $0.3 / 0.7$. This bi-exponential decay is in qualitative agreement with previous observations and has been attributed to intramolecular vibrational energy redistribution (IVR) between the symmetric and asymmetric stretching modes of the carbonyl ligands, $8,40,55,56$ but surface heterogeneity might contribute as well. Regarding the cross peak dynamics, we retrieve time constants for the vibrational energy transfer from ${ }^{13} \mathrm{CO}$ to ${ }^{12} \mathrm{CO}$ (upper cross peak in Figure 2) of about 90 ps on $\mathrm{TiO}_{2}, 110$ ps on ITO (which within error agrees with the result of Ref. ${ }^{8}, 90$ ps) and 260 ps on Au. Interestingly, the vibrational energy transfer on the plasmonic Au layers is even slower compared to that on dielectric substrates, despite the plasmonic enhancement of the absorption cross section. 


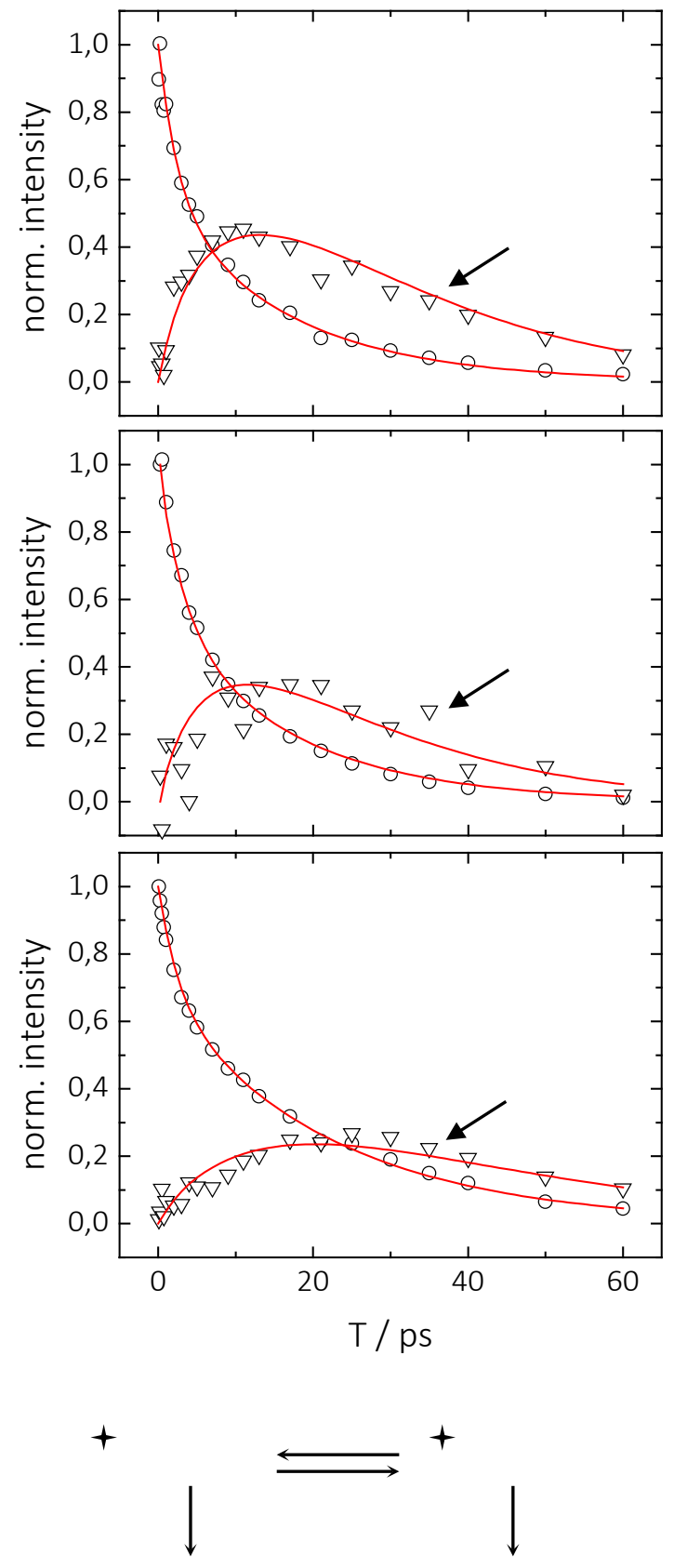

Figure 3. (a) - (c) Kinetic traces of ground state bleach signals of diagonal (circles) and cross peaks (triangles) together with kinetic fits (red lines) using the model from (d). Note that the cross peak signals are magnified by a factor of ten. (d) Schematic representation of vibrational energy transfer pathways and vibrational relaxation in the investigated sample systems. ET $\equiv$ energy transfer, bw $\equiv$ backward, fw $\equiv$ forward.

Evaluating Eq. 1, we have shown in our previous work that our preparation conditions result essentially in a closest possible packing of the molecules on ITO with intermolecular distances of about 4-5 $\AA .{ }^{8}$ In order to compare the results on the different substrates, we need to estimate the relative surface coverages, since the intermolecular distance strongly affects the transfer dynamics (Eq.1). We 
do this comparison here for $\mathrm{Au}$ and ITO, since the preparation of both materials by sputter-coating leads to the formation of nanoparticle layers with similar dimensions (see Fig. SI 2). Contrasting to that, $\mathrm{TiO}_{2}$ has been prepared by atomic-layer-deposition (ALD), likely revealing a more uniform layer. To determine the relative surface coverages on $\mathrm{Au}$ and ITO, we use the in-situ measured IR absorbance in conjunction with the experimentally determined enhancement factors. ${ }^{48,54}$ That is, the ATR IR absorbance for the Re carbonyl complexes on Au and ITO have been determined to about 36 mOD (Fig. SI 1 (a)) and 3.5 mOD (Fig. SI 1 (c)), while the corresponding enhancement factors are 30 and 2, respectively (Fig. SI 1 (a)-(d)). As the IR absorbance $\left(\mu^{2}\right)$ scales linearly with the enhancement factor EF, we obtain a surface coverage $\mathrm{c}$ on Au that is about 70\% of that on ITO. Eq. 1 predicts a $\mathrm{r}^{-6} \propto \mathrm{c}^{-3}$ scaling of the vibrational energy transfer rate, when assuming randomly distributed molecules on the surface without the formation of any domains. Based on the larger intermolecular distance, one would expect a three times slower transfer rate for the complexes on the Au layers as compared to ITO layers, in rough agreement with the experimental results (Figure 3). This larger distance is indeed expected for instance by considering that the binding motif of the S-Me linkers for the complexes on Au will be slightly different as compared to the $\mathrm{COOH}$ linkers at the bipyridyl ligand in case of oxide surfaces. Moreover, the increased sterical hindrance imposed by the methyl group is likely to result in a larger intermolecular spacing between the complexes.

This result, in turn, implies that plasmonic effects do not enhance the vibrational energy transfer rate. That is, if the signal enhancement measured for the Au sample, which scales as EF $\propto \mathrm{A} \propto \mu_{\text {eff }}^{2}$ (Eq. 2), would contribute to the transition dipole coupling as well, one would expect a $\mathrm{EF}^{2} \approx 230$ fold acceleration of the vibrational energy transfer rate relative to that on ITO (Eq. 1). This is clearly not observed; to the contrary, the vibrational energy rate goes down by a factor 2-3. Note that this is a huge discrepancy, which would safely overcompensate modest errors in the estimate of the intermolecular distance resulting from e.g. an inaccurate measurement in the surface coverage or the formation of domain of the surface. The same holds for variations of the orientational factor $\kappa$ in Eq. 1 , which might be smaller on $\mathrm{Au}$, since the molecules are oriented slightly differently due to the different geometry of the methyl-thioether linker.

Traditionally, the signal enhancement in surface spectroscopy is separated into an "electromagnetic" and a "chemical" contribution. ${ }^{47,48,57-63}$ The chemical enhancement mechanism takes into account substrate-adsorbate electronic interactions, resulting for instance in delocalized electronic states, charge-transfer states between the surface and the adsorbate, or change of electronic structure within the molecule. In essence, a chemical enhancement is the result of different partial charges within the molecule, which should enter the transition dipoles in Eqs. 1 and 2 in the same way. The absent speed-up of vibrational energy transfer on Au strongly suggests that such a 
chemical enhancement mechanism is not a very prominent effect here. That might indeed be expected, given the linker between the $\operatorname{Re}(\mathrm{CO})_{3}$-group and the surface, which decouples them electronically from each other.

Conversely, the electromagnetic mechanism ${ }^{61-66}$ originates from the polarizability of the metallic substrate. We start with noting that our Au surfaces are nano-structured (Fig. SI 2) with a plasmon resonance at about $625 \mathrm{~nm}$ (Fig. SI 3), while we investigate surface enhancements of vibrational transitions that are very far off-resonant in the mid-IR. ${ }^{48}$ We can therefore consider the electric field of the IR light as quasi-static, simplifying the discussion significantly. ${ }^{54}$ Figure 4 (a) illustrates two interacting dipoles $\vec{\mu}_{1}$ and $\vec{\mu}_{2}$ atop of a planar metal surface as the simplest possible geometry, together with their corresponding image-dipoles $\vec{\mu}_{\mathrm{i} 1}$ and $\vec{\mu}_{\mathrm{i} 2}$. For the absorption cross section of each one of these transition dipoles, both dipole and corresponding image dipole add up constructively, $\mu_{\text {eff }}=\mu+\mu_{\mathrm{i}}=2 \mu$, which according to Eq. 2 reveals an enhancement factor of $E F=4$.
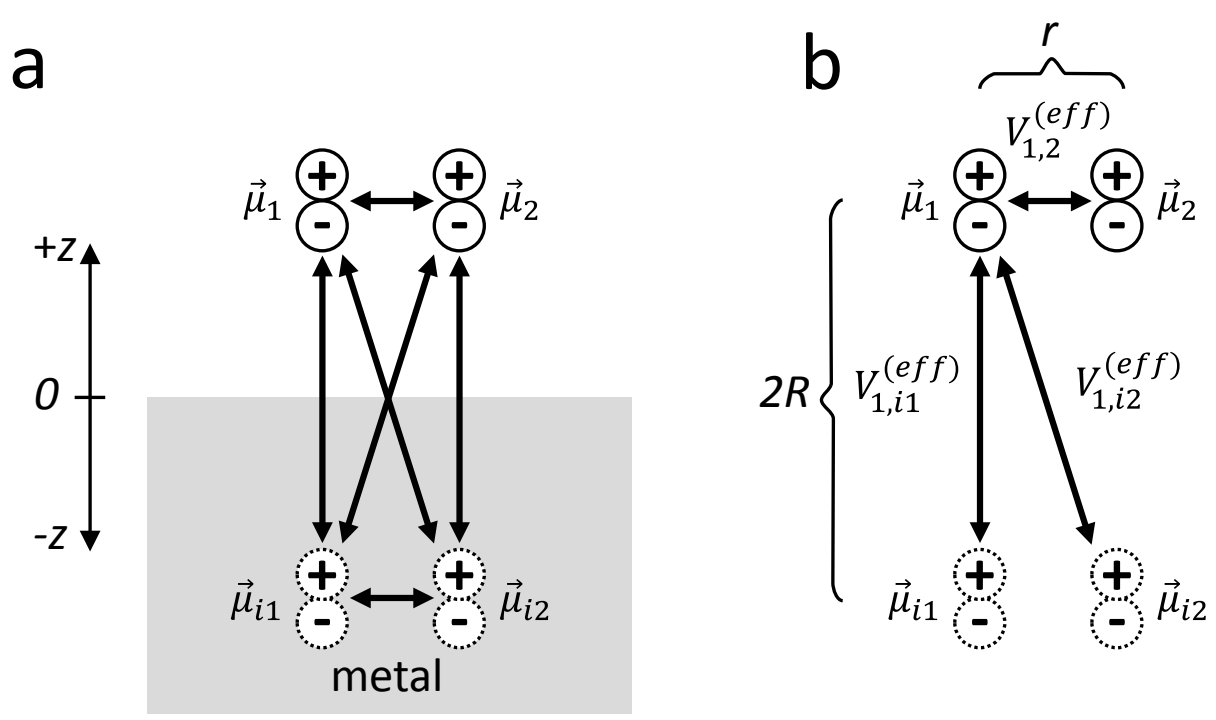

Figure 4. (a) Two interacting dipoles $\vec{\mu}_{1}$ and $\vec{\mu}_{2}$ atop of a metal surface, which we assume to be perpendicular to the surface, and which induce image dipoles $\vec{\mu}_{\mathrm{i} 1}$ and $\vec{\mu}_{\mathrm{i} 2}$. (b) In terms of interaction energies, this configuration can be reduced to one without metal and with only three remaining dipole-dipole interaction terms $V_{1,2}^{(\text {eff })}, V_{1,2 i}^{(\text {eff })}$, and $V_{1,1 i}^{(\text {eff) }}$ (see $\mathrm{SI}$ for the derivation). The separation of the two dipoles from the surface is $R$, and that among each other $r$.

With regard to the transition dipole coupling (Eq. 1), on the other hand, one can show that the configuration of Figure 4 (a) can be reduced to that of Figure 4 (b) without any metal and only two terms contributing to the coupling, namely the normal dipole-dipole interaction terms between the two transition dipoles $V_{1,2}^{(\text {eff })}$ as well as to the corresponding mirror transition dipole $V_{1,2 i}^{(e f f)}$ while somewhat non-intuitively, the coupling between the two image transition dipoles $\left(\mathrm{V}_{\mathrm{i} 1 \mathrm{i} 2 \mathrm{i}}\right)$ is lumped 
into $V_{1,2}^{(\text {eff })}$ and does not appear a second time (see $\mathrm{SI}$ for a detailed derivation). In simple words, this results from the fact that the electric field inside the metal vanishes, so $\vec{\mu}_{\mathrm{i} 1}$ cannot interact with $\vec{\mu}_{\mathrm{i} 2}$. In our concrete case, the distance between two dipoles exhibiting vibrational energy transfer is $r=4-5$ $\AA^{8}{ }^{8}$ while the distance of the CO groups to the surface is $R \approx 10 \AA$, hence the distance to the image dipole is $2 R \approx 20 \AA$, about four times larger than the intermolecular distance. Since the transition dipole coupling scales as $\mathrm{r}^{-3}$, we may safely neglect $\mathrm{V}_{1,2 \mathrm{i}}^{(\mathrm{eff})}$ and find that the transition dipole coupling is determined by $V_{1,2}^{(\text {eff) }}$ only, as if the metal surface would not be present. That derivation explains why we do not observe any acceleration of the vibrational energy transfer rate despite the fact that we do observe an enhancement of the absorption.

Note that Figure 4 discusses the situation on a perfectly planar surface, in which case an enhancement factor of $E F=4$ is expected for absorption. That enhancement factor is obtained as long as the dipole approximation holds for the total dipole $\mu_{\text {eff }}$, i.e., whenever the distance of the dipole from its image dipole $2 R$ is much smaller than the wavelength of the light (which we can indeed safely assume). The enhancement factor is larger in our concrete experiment (EF $\approx 30)$ due to the roughness of the surface and possibly hotspots between Au nanoparticles on the surface. ${ }^{48}$ We expect that the argument of Figure 4 remains in essence the same, however, in that case it is the roughness of the surface, as characterized by the typical curvature radius, which determines the distance from the surface below which the enhancement factor is large. ${ }^{54}$

The IR enhancement from the Au layers, which we have used here, are rather small $(<100)$ as compared to other previous studies. ${ }^{49,67-70}$ In this context, particularly purposely tailored nanostructures such as nano-antennae or nano-slits with plasmon resonances located directly in the mid-IR spectral range have been shown to dramatically enhance IR absorbances. ${ }^{67,69-74}$ In that case, the quasi-static approximation, which we used in the derivation of Figure 4, no longer holds. It is not a priori clear if the conclusions drawn here are generalizable to such substrates as well. The spectral overlap between the intense and tuneable plasmon resonance and the molecular IR resonance could indeed result in strong coupling between the two, thus potentially affecting the transition dipole coupling between adsorbates in a meaningful way. It would thus be important to compare the results presented here to analogous experiments on engineered arrays of nano-antennae.

Among the previous 2D IR investigations of Re-carbonyl complexes on various types of surfaces, $18,20,24,25,38-45$ the studies by Xiong et al. ${ }^{42,43}$ are the most closely related to our present report. That works has specifically looked at substrate-adsorbate interactions between an immobilized Re-complex and an underlying Au surface, suggesting that dipole/image-dipole interactions cause severe differences in lineshapes on the same molecule. ${ }^{42,43}$ As a key result, it was found that out of three 
carbonyl modes (i.e. $A^{\prime}(1), A^{\prime}(2)$ and $\left.A^{\prime \prime}\right)$, the $A^{\prime}(1)$ mode has the largest surface-distance and thus experiences the smallest interaction with its image-dipoles, in very good agreement with the results and interpretation presented here.

In summary, we have shown that vibrational energy transfer between surface-bound rhenium carbonyl complexes is not promoted on plasmonic substrates, despite the fact that the absorption cross section is significantly enhanced. In other words, the effective transition dipoles entering Eqs. 1 and 2 are not the same. By comparing vibrational energy transfer between the symmetric carbonyl stretching vibrations on three different substrates, we find that the rate is even slightly slower on plasmonic metal layers as compared to purely dielectric layers, while they are about the same on the oxides $\mathrm{TiO}_{2}$ and ITO. The transition dipole and its corresponding image dipole add up constructively for the absorption cross section (Eq. 2) on a length scale that is determined by roughness of the surface. On the contrary, the very small "Förster" radius for vibrational energy transfer in essence supresses the dipole coupling to the image dipole for the purpose of vibrational energy transfer (Eq.1). This result, in turn, allows one to use the vibrational energy transfer rate as a measure to determine intermolecular distances on a surface, regardless of its plasmonic properties. Both linker and surface chemistry are about the same in our $\mathrm{TiO}_{2}$ and ITO preparations, and consequently also the vibrational energy transfer rates, while vibrational energy transfer is somewhat slower on gold, presumably due to the different structures of the methyl-thioether linker group. Similar experiments on purposely designed plasmonic nanostructures, which are resonant with the IR transition and consequently reveal significantly larger enhancement factors, will be most interesting to see whether the conclusions drawn here can be generalized to those structures.

\section{Experimental}

2D ATR IR experiments were performed using the setup described in detail in refs. ${ }^{35,36}$ In brief, IR pump and probe pulses are derived from an optical parametric amplifier and are spatially overlapped at the reflecting plane of a single reflection $\mathrm{CaF}_{2}$ ATR element. Behind the sample, the probe beam is detected with a 32-pixel MCT detector. Absorptive 2D IR spectra are collected by scanning the coherence delay between two pump pulses in a Mach-Zehnder interferometer for a fixed population delay. The polarization of all IR beams is in the plane of the prism surface (s-polarized).

The $\mathrm{CaF}_{2}$ ATR prisms are coated with $\mathrm{TiO}_{2}$, ITO and $\mathrm{Au}$ via different methods. In case of $\mathrm{TiO}_{2}$, atomic layer deposition (ALD) is employed using tetrakis(dimethylamido)titanium(IV) (99.999\%, SigmaAldrich) and deionized water as precursor materials. The ALD system used is a Picosun RS 200. The deposition is performed at $120^{\circ} \mathrm{C}$ for 380 cycles. The Ti precursor (heated to $85^{\circ} \mathrm{C}$ ) is pulsed for $1.6 \mathrm{~s}$ 
(using the software boost function), followed by a $6.0 \mathrm{~s} \mathrm{~N}_{2}$ purge. $\mathrm{H}_{2} \mathrm{O}$ (at room temperature) is then pulsed for $0.1 \mathrm{~s}$ pulse, followed by a $6.0 \mathrm{~s} \mathrm{~N} \mathrm{~N}_{2}$ purge. The thickness of the film $(21 \mathrm{~nm})$ is determined using ellipsometry on a $2 \times 2 \mathrm{~cm}$ piece of silicon wafer added to the reaction chamber at the time of deposition. In case of ITO and Au, we use the sputter-coating technique in a Bal-tec SCD 500 sputter coater (Leica Microsystems, Vienna, Austria). The thickness of the films is in-situ determined by use of a quartz microbalance. The sputtering process was conducted at a working distance of $50 \mathrm{~mm}$, a pressure of $0.8 \times 10-5 \mathrm{mbar}$, an Ar pressure of $0.1 \mathrm{mbar}$, and a current of $30 \mathrm{~mA}$ (for ITO), or $12 \mathrm{~mA}$ (for $\mathrm{Au}$ ). This resulted in a deposition rate of about $0.02 \mathrm{~nm} \mathrm{~s}^{-1}$ for both materials. Here, we use average layer thicknesses of $5 \mathrm{~nm}$ ITO and $0.5 \mathrm{~nm}$ Au. The very thin layer of Au was chosen to avoid lineshape distortions in the 2D ATR IR signals. ${ }^{35}$

As the $\mathrm{TiO}_{2}$ ALD was carried out at relatively low temperature, the films are amorphous. ${ }^{75}$ Although the precise surface structure of the amorphous ALD $\mathrm{TiO}_{2}$ is unknown, theoretical work suggests a local structure similar to that of crystalline $\mathrm{TiO}_{2},{ }^{76}$ and we assume a similar binding of the molecular catalysts to this amorphous layer.

The sample molecules $\operatorname{Re}\left({ }^{13} \mathrm{CO}\right)_{3} \mathrm{Br}$ and $\operatorname{Re}\left({ }^{12} \mathrm{CO}\right)_{3} \mathrm{Cl}$ were prepared according to previously published procedures.,77 4,4'-dimethylthio-2,2'-bipyridine and 2,2'-bipyridine-4,4'-dicarboxylic acid were purchased from Carbosynth (Berkshire, UK) and Sigma-Aldrich $(\mathrm{CH})$, respectively, and used without further purification. Mixtures of $\operatorname{Re}\left({ }^{13} \mathrm{CO}\right){ }_{3} \mathrm{Br} / \operatorname{Re}\left({ }^{12} \mathrm{CO}\right){ }_{3} \mathrm{Cl}$ were then dissolved in methanol $(\mathrm{MeOH})$ to a concentration of $0.1 \mathrm{mM}$ for the preparation of the monolayer samples. To adsorb the molecules on the surfaces, the $\mathrm{TiO}_{2} / \mathrm{ITO} / \mathrm{Au}$-coated prisms were immersed overnight in freshly prepared methanolic solutions of $\operatorname{Re}\left({ }^{13} \mathrm{CO}\right){ }_{3} \mathrm{Br} / \operatorname{Re}\left({ }^{12} \mathrm{CO}\right)_{3} \mathrm{Cl}$, then thoroughly washed with $\mathrm{MeOH}$ and sonicated for 10 minutes in $\mathrm{MeOH}$ environment. During the measurements, the samples were then also kept in $\mathrm{MeOH}$ environment.

\section{Acknowledgements}

We would like to thank Michael D. Fayer, Martin T. Zanni and Madhavi Krishnan for stimulating comments on energy transfer mechanisms and for most insightful discussions. We furthermore acknowledge the experimental support by Rolf Pfister, Angelo Frei and Roger Alberto regarding the synthesis of the investigated samples. Experimental support by Andres Kaech from the Center of Microscopy and Imaging at the University of Zurich (ZMB) with regard to the preparation and characterization of the substrate layers is also gratefully acknowledged. This research was funded in part by the Swiss National Science Foundation through grant number CRSII2_160801/1, AP Energy Grant \# PYAPP2 160586, and by the URPP LightChEC of the University of Zurich. 


\section{Supporting Information:}

Determination of Enhancement Factor on $\mathrm{Au}$, ITO and $\mathrm{TiO}_{2}$, surface characterization of ITO and $\mathrm{Au}$ layers by use of Scanning Electron Microscopy, spectral characterization of the plasmon resonance in $\mathrm{Au}$ layers, and the theory of the coupling of two dipoles on a metal surface. This information is available free of charge via the Internet at http://pubs.acs.org.

\section{Author Information}

*philip.kraack@gmail.com

Phone: +414463544 77

Fax: +41446356838

‡peter.hamm@chem.uzh.ch

Phone: +41 446354431

Fax: +41 446356838 


\section{References}

(1) Mirkovic, T.; Ostroumov, E. E.; Anna, J. M.; van Grondelle, R.; Govindjee; Scholes, G. D. Light Absorption and Energy Transfer in the Antenna Complexes of Photosynthetic Organisms. Chem. Rev. 2017, 117, 249-293.

(2) Scholes, G. D. Long-Range Resonance Energy Transfer in Molecular Systems. Annu. Rev. Phys. Chem. 2003, 54, 57-87.

(3) Chenu, A.; Scholes, G. D. Coherence in Energy Transfer and Photosynthesis. Annu. Rev. Phys. Chem. 2014, 66, 69-96.

(4) Chen, H.; Bian, H.; Li, J.; Wen, X.; Zheng, J. Ultrafast Multiple-Mode Multiple-Dimensional Vibrational Spectroscopy. Int. Rev. Phys. Chem. 2012, 31, 469-565.

(5) Neuhaus, D.; Williamson, M. P. The Nuclear Overhauser Effect in Structural and Conformational Analysis; VCH New York: New York, 1989.

(6) van der Meer, B. W. Förster Theory. In FRET - Förster Resonance Energy Transfer; Wiley-VCH Verlag GmbH \& Co. KGaA, 2013; pp. 23-62.

(7) Ostrander, J. S.; Knepper, R.; Tappan, A. S.; Kay, J. J.; Zanni, M. T.; Farrow, D. A. Energy Transfer Between Coherently Delocalized States in Thin Films of the Explosive Pentaerythritol Tetranitrate (PETN) Revealed by Two-Dimensional Infrared Spectroscopy. J. Phys. Chem. B 2017, 121, 1352-1361.

(8) Kraack, J. P.; Frei, A.; Alberto, R.; Hamm, P. Ultrafast Vibrational Energy-Transfer in Catalytic Monolayers at Solid-Liquid Interfaces. J. Phys. Chem. Lett. 2017, 8, 2489-2495.

(9) Chen, H.; Wen, X.; Guo, X.; Zheng, J. Intermolecular Vibrational Energy Transfers in Liquids and Solids. Phys. Chem. Chem. Phys. 2014, 16, 13995-14014.

(10) Kraack, J. P.; Kaech, A.; Hamm, P. Molecule-Specific Interactions of Diatomic Adsorbates at Metal-Liquid Interfaces. Struct. Dyn. 2017, 4, 044009 1-14.

(11) Zhang, Z.; Piatkowski, L.; Bakker, H. J.; Bonn, M. Ultrafast Vibrational Energy Transfer at the Water/air Interface Revealed by Two-Dimensional Surface Vibrational Spectroscopy. Nat. Chem. 2011, 3, 888-893.

(12) Piatkowski, L.; Eisenthal, K. B.; Bakker, H. J. Ultrafast Intermolecular Energy Transfer in Heavy Water. Phys. Chem. Chem. Phys. 2009, 11, 9033-9038.

(13) Woutersen, S.; Bakker, H. J. Resonant Intermolecular Transfer of Vibrational Energy in Liquid 
Water. Nature 1999, 402, 507-509.

(14) Woutersen, S.; Mu, Y.; Stock, G.; Hamm, P. Subpicosecond Conformational Dynamics of Small Peptides Probed by Two-Dimensional Vibrational Spectroscopy. Proc. Natl. Acad. Sci. U. S. A. 2001, 98, 11254-11258.

(15) Bonn, M.; Hess, C.; Wolf, M. The Dynamics of Vibrational Excitations on Surfaces: CO on Ru(001). J. Chem. Phys. 2001, 115, 7725-7735.

(16) Ulman, A. Formation and Structure of Self-Assembled Monolayers. Chem. Rev. 1996, 96, 15331554.

(17) Love, J. C.; Estroff, L. A.; Kriebel, J. K.; Nuzzo, R. G.; Whitesides, G. M. Self-Assembled Monolayers of Thiolates on Metals as a Form of Nanotechnology. Chem. Rev. 2005, 105, 11031169.

(18) Rosenfeld, D. E.; Gengeliczki, Z.; Smith, B. J.; Stack, T. D. P.; Fayer, M. D. Structural Dynamics of a Catalytic Monolayer Probed by Ultrafast 2D IR Vibrational Echoes. Science 2011, 334, 634639.

(19) Kraack, J. P.; Lotti, D.; Hamm, P. 2D Attenuated Total Reflectance Infrared Spectroscopy Reveals Ultrafast Vibrational Dynamics of Organic Monolayers at Metal-Liquid Interfaces. J. Chem. Phys. 2015, 142, 212413.

(20) Yan, C.; Yuan, R.; Nishida, J.; Fayer, M. D. Structural Influences on the Fast Dynamics of Alkylsiloxane Monolayers on SiO 2 Surfaces Measured with 2D IR Spectroscopy. J. Phys. Chem. C 2015, 119, 16811-16823.

(21) Rosenfeld, D. E.; Fayer, M. D. Excitation Transfer Induced Spectral Diffusion and the Influence of Structural Spectral Diffusion. J. Chem. Phys. 2012, 137, 064109 1-18.

(22) Li, J.; Qian, H.; Chen, H.; Zhao, Z.; Yuan, K.; Chen, G.; Miranda, A.; Guo, X.; Chen, Y.; Zheng, N.; et al. Two Distinctive Energy Migration Pathways of Monolayer Molecules on Metal Nanoparticle Surfaces. Nat. Commun. 2016, 7, 10749.

(23) Li, J.; Chen, H.; Miranda, A.; Yuan, K.; Chen, Y.; Shen, Y.; Jiang, B.; Zhang, Y.; Guo, X.; Zheng, J. Non-Resonant Vibrational Energy Transfer on Metal Nanoparticle/Liquid Interface. J. Phys. Chem. C 2016, 120, 25173-25179.

(24) Oudenhoven, T. A.; Joo, Y.; Laaser, J. E.; Gopalan, P.; Zanni, M. T. Dye Aggregation Identified by Vibrational Coupling Using 2D IR Spectroscopy. J. Chem. Phys. 2015, 142, 212449 1-12. 
(25) Laaser, J. E.; Christianson, R.; Oudenhoven, T. A.; Joo, Y.; Gopalan, P.; Schmidt, J. R.; Zanni, M. T. Dye Self-Association Identified by Intermolecular Couplings between Vibrational Modes As Revealed by Infrared Spectroscopy, and Implications for Electron Injection. J. Phys. Chem. C $2014,118,5854-5861$

(26) Zamadar, M.; Asaoka, S.; Grills, D. C.; Miller, J. R. Giant Infrared Absorption Bands of Electrons and Holes in Conjugated Molecules. Nat. Commun. 2013, 4, 2818.

(27) Sakamoto, A.; Harada, T.; Tonegawa, N. A New Approach to the Spectral Study of Unstable Radicals and Ions in Solution by the Use of an Inert Gas Glovebox System: Observation and Analysis of the Infrared Spectra of the Radical Anion and Dianion of P-Terphenyl. J. Phys. Chem. A 2008, 112, 1180-1187.

(28) Baiblch, I. M.; English, A. M.; Butler, I. S. Absolute Integrated Infrared Intensities of the Carbonyl, Thlocarbonyl, and Selenocarbonyl Stretching Modes in the Chalcocarbonyl Complexes $\mathrm{Cr}(\mathrm{CO}) 5(\mathrm{CX})$ and $(n 6 \mathrm{C} 6 \mathrm{H} 6) \mathrm{Cr}(\mathrm{CO}) 2(\mathrm{CX})(\mathrm{X}=\mathrm{O}, \mathrm{S}$, Se). Organometallics 1984, 3, 17861789.

(29) Moskovits, M. Surface Selection Rules. J. Chem. Phys. 1982, 77, 4408-4416.

(30) Ding, S.-Y.; You, E.-M.; Tian, Z.-Q.; Moskovits, M. Electromagnetic Theories of Surface-Enhanced Raman Spectroscopy. Chem. Soc. Rev. 2017, 46, 4042-4076.

(31) Moskovits, M. Surface-Enhanced Spectroscopy. Rev. Mod. Phys. 1985, 57, 783-826.

(32) Aroca, R. Surface-Enhanced Vibrational Spectroscopy; John Wiley \& Sons, Ltd: Chichester, UK, 2006.

(33) Hamm, P.; Zanni, M. T. Concepts and Methods of 2D Infrared Spectroscopy; Cambridge University Press: New York, 2011.

(34) Kraack, J. P. Ultrafast Structural Molecular Dynamics Investigated with 2D Infrared Spectroscopies. Top Curr Chem 2017, 375:86, 1-93.

(35) Kraack, J. P.; Hamm, P. Surface-Sensitive and Surface-Specific Ultrafast Two-Dimensional Vibrational Spectroscopy. Chem. Rev. 2017, 117, 10623-10664.

(36) Kraack, J. P.; Lotti, D.; Hamm, P. Ultrafast, Multidimensional Attenuated Total Reflectance Spectroscopy of Adsorbates at Metal Surfaces. J. Phys. Chem. Lett. 2014, 5, 2325-2329.

(37) Kraack, J. P.; Lotti, D.; Hamm, P. Surface-Enhanced, Multi-Dimensional Attenuated Total Reflectance Spectroscopy. In Proc. of SPIE, Physical Chemistry of Interfaces and Nanomaterials 
XIV; Sophia C. Hayes; Eric R. Bittner, Eds.; 2015; Vol. 9549, p. 95490 S.

(38) Vanselous, H.; Stingel, A. M.; Petersen, P. B. Interferometric 2D Sum Frequency Generation Spectroscopy Reveals Structural Heterogeneity of Catalytic Monolayers on Transparent Materials. J. Phys. Chem. Lett. 2017, 8, 825-830.

(39) Xiong, W.; Laaser, J. E.; Paoprasert, P.; Franking, R. A.; Hamers, R. J.; Gopalan, P.; Zanni, M. T. Transient 2D IR Spectroscopy of Charge Injection in Dye-Sensitized Nanocrystalline Thin Films. J. Am. Chem. Soc. 2009, 131, 18040-18041.

(40) Anfuso, C. L.; Ricks, A. M.; Rodr, W.; Lian, T. Ultrafast Vibrational Relaxation Dynamics of a Rhenium Bipyridyl. J. Phys. Chem. C 2012, 116, 26377-26384.

(41) Calabrese, C.; Vanselous, H.; Petersen, P. B. Deconstructing the Heterogeneity of SurfaceBound Catalysts: Rutile Surface Structure Affects Molecular Properties. J. Phys. Chem. C 2016, 120, 1515-1522.

(42) Li, Y.; Wang, J.; Clark, M. L.; Kubiak, C. P.; Xiong, W. Characterizing Interstate Vibrational Coherent Dynamics of Surface Adsorbed Catalysts by Fourth-Order 3D SFG Spectroscopy. Chem. Phys. Lett. 2016, 650, 1-6.

(43) Wang, J.; Clark, M. L.; Li, Y.; Kaslan, C. L.; Kubiak, C. P.; Xiong, W. Short-Range Catalyst-Surface Interactions Revealed by Heterodyne Two-Dimensional Sum Frequency Generation Spectroscopy. J. Phys. Chem. Lett. 2015, 6, 4204-4209.

(44) Rosenfeld, D. E.; Nishida, J.; Yan, C.; Kumar, S. K. K.; Tamimi, A.; Fayer, M. D. Structural Dynamics at Monolayer-Liquid Interfaces Probed by 2D IR Spectroscopy. J. Phys. Chem. C 2013, $117,1409-1420$.

(45) Yan, C.; Yuan, R.; Pfalzgraff, W. C.; Nishida, J.; Wang, L.; Markland, T. E.; Fayer, M. D. Unraveling the Dynamics and Structure of Functionalized Self-Assembled Monolayers on Gold Using 2D IR Spectroscopy and MD Simulations. Proc. Natl. Acad. Sci. 2016, 113, 4929-4934.

(46) Yan, C.; Thomaz, J. E.; Wang, Y.-L.; Nishida, J.; Yuan, R.; Breen, J. P.; Fayer, M. D. Ultrafast to Ultraslow Dynamics of a Langmuir Monolayer at the Air/Water Interface Observed with Reflection Enhanced 2D IR Spectroscopy. J. Am. Chem. Soc. 2017, 139, 16518-16527.

(47) Osawa, M. Surface-Enhanced Infrared Absorption. In Near-Field Optics and Surface Plasmon Polaritons; S. Kawata, Ed.; Springer-Verlag Berlin Heidelberg, 2001; Vol. 81, pp. 163-187.

(48) Kraack, J. P.; Kaech, A.; Hamm, P. Surface Enhancement in Ultrafast 2D ATR IR Spectroscopy at the Metal-Liquid Interface. J. Phys. Chem. C 2016, 120, 3350-3359. 
(49) Kraack, J. P.; Hamm, P. Vibrational Ladder-Climbing in Surface-Enhanced, Ultrafast Infrared Spectroscopy. Phys. Chem. Chem. Phys. 2016, 18, 16088-16093.

(50) Lotti, D.; Hamm, P.; Kraack, J. P. Surface-Sensitive Spectro-Electrochemistry Using Ultrafast 2D ATR IR Spectroscopy. J. Phys. Chem. C 2016, 120, 2883-2892.

(51) Franzen, S.; Rhodes, C.; Cerruti, M.; Gerber, R. W.; Losego, M.; Maria, J.-P.; Aspnes, D. E. Plasmonic Phenomena in Indium Tin Oxide and ITO-Au Hybrid Films. Opt. Lett. 2009, 34, 28672869.

(52) Ma, K.; Zhou, N.; Yuan, M.; Li, D.; Yang, D. Tunable Surface Plasmon Resonance Frequencies of Monodisperse Indium Tin Oxide Nanoparticles by Controlling Composition, Size, and Morphology. Nanoscale Res. Lett. 2014, 9, 547.

(53) Kim, J.; Naik, G. V.; Emani, N. K.; Guler, U.; Boltasseva, A. Plasmonic Resonances in Nanostructured Transparent Conducting Oxide Films. IEEE J. Sel. Top. Quantum Electron. 2013, $19,1-7$.

(54) Donaldson, P. M.; Hamm, P. Gold Nanoparticle Capping Layers: Structure, Dynamics, and Surface Enhancement Measured Using 2D-IR Spectroscopy. Angew. Chemie-International Ed. $2013,52,634-638$.

(55) Ricks, A. M.; Anfuso, C. L.; Rodríguez-Córdoba, W.; Lian, T. Vibrational Relaxation Dynamics of Catalysts on TiO2 Rutile (1\&\#xa0;1\&\#xa0;0) Single Crystal Surfaces and Anatase Nanoporous Thin Films. Chem. Phys. 2013, 422, 264-271.

(56) Delor, M.; Sazanovich, I. V.; Towrie, M.; Spall, S. J.; Keane, T.; Blake, A. J.; Wilson, C.; Meijer, A. J. H. M.; Weinstein, J. A. Dynamics of Ground and Excited State Vibrational Relaxation and Energy Transfer in Transition Metal Carbonyls. J. Phys. Chem. B 2014, 118, 11781-11791.

(57) Lecomte, S.; Matejka, P.; Baron, M. H. Correlation between Surface Enhanced Raman Scattering and Absorbance Changes in Silver Colloids . Evidence for the Chemical Enhancement Mechanism. Langmuir 1998, 7463, 4373-4377.

(58) Otto, A. The "Chemical" (Electronic) Contribution to Surface-Enhanced Raman Scattering. J. Raman Spectrosc. 2005, 36, 497-509.

(59) Jayawardhana, S.; Rosa, L.; Juodkazis, S.; Stoddart, P. R. Additional Enhancement of Electric Field in Surface-Enhanced Raman Scattering due to Fresnel Mechanism. Sci. Rep. 2013, 3, 2335.

(60) Saikin, S. K.; Chu, Y.; Rappoport, D.; Crozier, K. B.; Aspuru-Guzik, A. Separation of Electromagnetic and Chemical Contributions to Surface-Enhanced Raman Spectra on 
Nanoengineered Plasmonic Substrates. J. Phys. Chem. Lett. 2010, 1, 2740-2746.

(61) Osawa, M.; Ataka, K. Electromagnetic Mechanism of Enhanced Infrared Absorption of Molecules Adsorbed on Metal Island Films. Surf. Sci. Lett. 1992, 262, L118-L122.

(62) Alonso-González, P.; Albella, P.; Schnell, M.; Chen, J.; Huth, F.; García-Etxarri, A.; Casanova, F.; Golmar, F.; Arzubiaga, L.; Hueso, L. E.; et al. Resolving the Electromagnetic Mechanism of Surface-Enhanced Light Scattering at Single Hot Spots. Nat. Commun. 2012, 3, 684.

(63) Schatz, G. C.; van Duyne, R. P. Electromagnetic Mechanism of Surface-Enhanced Spectroscopy. In Handbook of Vibrational Spectroscopy; John Wiley \& Sons, Ltd.: Chichester, UK, 2002; pp. 116.

(64) Novotny, L.; van Hulst, N. Antennas for Light. Nat. Photonics 2011, 5, 83-90.

(65) Novotny, L. From near-Field Optics to Optical Antennas Feature. Phys. Today 2011, 47-52.

(66) Novotny, L.; Hecht, B. Principles of Nano-Optics; 1st ed.; Cambridge University Press: New York, 2006

(67) Selig, O.; Siffels, R.; Rezus, Y. L. A. Ultrasensitive Ultrafast Vibrational Spectroscopy Employing the Near Field of Gold Nanoantennas. Phys. Rev. Lett. 2015, 114, 233004 1-5.

(68) Enders, D.; Nagao, T.; Pucci, A.; Nakayama, T.; Aono, M. Surface-Enhanced ATR-IR Spectroscopy with Interface-Grown Plasmonic Gold-Island Films near the Percolation Threshold. Phys. Chem. Chem. Phys. 2011, 13, 4935-4941.

(69) Huck, C.; Vogt, J.; Sendner, M.; Hengstler, D.; Neubrech, F.; Pucci, A. Plasmonic Enhancement of Infrared Vibrational Signals: Nanoslits versus Nanorods. ACS Photonics 2015, 2, 1489-1497.

(70) Neubrech, F.; Pucci, A. Plasmonic Enhancement of Vibrational Excitations in the Infrared. IEEE J. Sel. Top. Quantum Electron. 2013, 19, 4600809-4600809.

(71) Adato, R.; Aksu, S.; Altug, H. Engineering Mid-Infrared Nanoantennas for Surface Enhanced Infrared Absorption Spectroscopy. Mater. Today 2015, 18, 436-446.

(72) Wu, C.; Khanikaev, A. B.; Adato, R.; Arju, N.; Yanik, A. A.; Altug, H.; Shvets, G. Fano-Resonant Asymmetric Metamaterials for Ultrasensitive Spectroscopy and Identification of Molecular Monolayers. Nat. Mater. 2012, 11, 69-75.

(73) Rodrigo, D.; Limaj, O.; Janner, D.; Etezadi, D.; Abajo, F. J. G. de; Pruneri, V.; Altug, H. MidInfrared Plasmonic Biosensing with Graphene. Science 2016, 349, 165-168. 
(74) Lal, S.; Grady, N. K.; Kundu, J.; Levin, C. S.; Lassiter, J. B.; Halas, N. J. Tailoring Plasmonic Substrates for Surface Enhanced Spectroscopies. Chem. Soc. Rev. 2008, 37, 898-911.

(75) Azevedo, J.; Steier, L.; Dias, P.; Stefik, M.; Sousa, C. T.; Araújo, J. P.; Mendes, A.; Graetzel, M.; Tilley, S. D. On the Stability Enhancement of Cuprous Oxide Water Splitting Photocathodes by Low Temperature Steam Annealing. Energy Environ. Sci. 2014, 7, 4044-4052.

(76) Prasai, B.; Cai, B.; Underwood, M. K.; Lewis, J. P.; Drabold, D. A. Properties of Amorphous and Crystalline Titanium Dioxide from First Principles. J. Mater. Sci. 2012, 47, 7515-7521.

(77) Frei, A.; Sidler, D.; Mokolokolo, P.; Braband, H.; Fox, T.; Spingler, B.; Roodt, A.; Alberto, R. Kinetics and Mechanism of CO Exchange in Fac -[MBr 2 (solvent)(CO) 3 ] - (M = Re, 99 Tc). Inorg. Chem. 2016, 55, 9352-9360. 